\title{
Operational Experience with the ATLAS Pixel Detector
}

\author{
Clemens Lange, Member, IEEE, On behalf of the ATLAS Collaboration.
}

\begin{abstract}
The ATLAS Pixel Detector is the innermost part of the ATLAS Detector which is operated at the Large Hadron Collider (LHC) at CERN. It is a three-hit tracking system that provides high-resolution measurements of charged particle tracks and secondary vertices that are essential to the physics goals of ATLAS. The detector consists of 1744 silicon sensors totalling approximately 80 million electronic channels. The system operated smoothly with very high efficiency. In this paper operational experience with the Pixel Detector will be presented, including monitoring and detector calibration procedures. In addition, the current status of the Pixel Detector and its response to LHC high energy proton-proton collisions are presented.
\end{abstract}

Index Terms-ATLAS, pixel detector, operation, LHC

\section{INTRODUCTION}

$\mathbf{T}$ HE ATLAS detector encloses the interaction region located at point 1 of the Large Hadron Collider (LHC) ring at CERN and is one of two general purpose experiments constructed to discover the Higgs boson and to search for new physics at high energies [1]. At design parameters, the LHC will collide bunches of $1.2 \times 10^{11}$ protons every $25 \mathrm{~ns}$ at a centre of mass energy of $14 \mathrm{TeV}$ for an expected instantaneous luminosity of $10^{34} \mathrm{~cm}^{-2} \mathrm{~s}^{-1}$. The ATLAS detector was designed to operate in an environment with this high rate of interactions, maintaining high efficiency and excellent resolution as well as good particle identification. To accomplish this, the detector is designed in an onion-like structure that comprises three sub-systems: The Inner Detector (ID) consists of the Pixel Detector, the Semi-Conductor Tracker and the Transition Radiation Tracker, immersed in a $2 \mathrm{~T}$ solenoid magnetic field. It is responsible for tracking and particle identification. Surrounding the ID, calorimeter systems are placed that measure the energy of the particles created in the collision and subsequent decays. The muon system constitutes the outermost layer with large superconducting air-core barrel loops generating a toroidal field of $4 \mathrm{~T}$.

This paper focusses on the ATLAS Pixel Detector [2] and specifically on details relating to the operation of the detector during the first two years of LHC running. The technologies employed are described, along with the properties of the detector. Special attention is given to the calibration and tuning of the system, followed by a discussion of the operational characteristics of the detector and the consequences of running in an environment of high radiation.

Manuscript received November 21, 2011.

C. Lange is with Deutsches Elektronen-Synchrotron (DESY), Platanenallee 6,15738 Zeuthen, Germany (e-mail: clemens.lange@ desy.de).

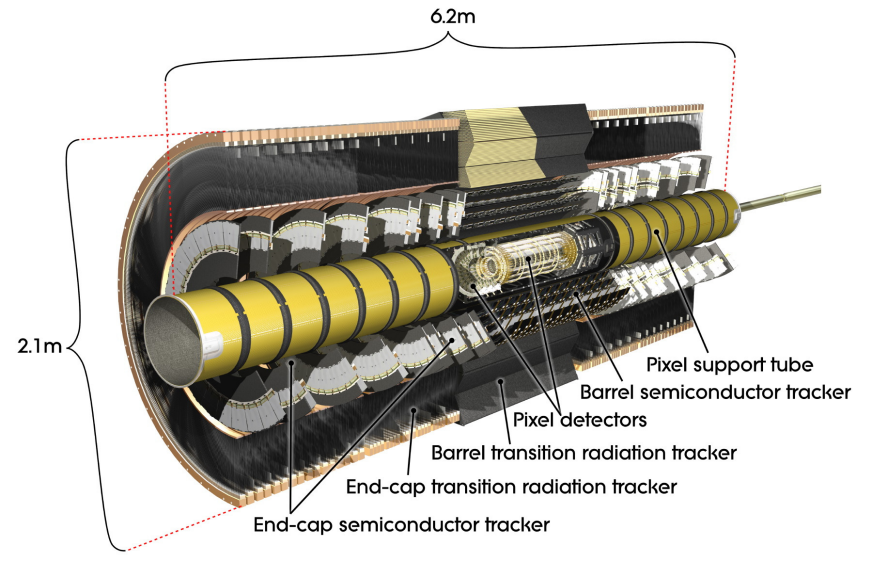

Fig. 1. The ATLAS Pixel Detector [2].

\section{The ATLAS Pixel Detector}

\section{A. Layout and structure}

Designed to operate in the high particle multiplicity of LHC, the ATLAS Pixel Detector has a position resolution in the $r-\phi$ plane of $<15 \mu \mathrm{m}$, as well as a time resolution of less than the 25 ns collision rate of the LHC. Built as a three-hit system for the central region of the ATLAS detector (pseudo-rapidity $|\eta|<2.5)$, it consists of three cylindrical barrel layers with 1456 modules and two end-caps with three disks each having a total of 288 modules as depicted in Fig. 1. The barrel layers have radii of $50.5 \mathrm{~mm}, 88.5 \mathrm{~mm}$ and $122.5 \mathrm{~mm}$ and are 800 $\mathrm{mm}$ long.

The modules need to be cooled to prevent overheating and reverse annealing of the silicon sensors. Therefore, evaporative $C_{3} F_{8}$ cooling is integrated into the support structure and an average temperature of $-13^{\circ} \mathrm{C}$ is achieved.

\section{B. The ATLAS Pixel Detector module}

A pixel module consists of a $250 \mu \mathrm{m}$ thick sensor with $n^{+}$ pixels implanted on the $n$-doped bulk with a $p^{+}$backplane, 16 Front-End chips (FE-I3) and a module controller chip (MCC) [4]. The FE-I3 chips [3] were manufactured in radiation tolerant $0.25 \mu \mathrm{m}$ CMOS technology and have 2880 readout channels. Each module has an active area of $16.4 \times 60.8 \mathrm{~mm}^{2}$ consisting of $47232(328 \times 144)$ pixels. The typical pixel size is $50 \times 400 \mu \mathrm{m}^{2}$. To enable full coverage in the regions between front-end chips, approximately $10 \%$ of the sensor pixels have a size of $600 \times 50 \mu \mathrm{m}^{2}$ (long pixels). In order to fully deplete the semi-conductor a bias voltage 150 to $600 \mathrm{~V}$ can be applied. 


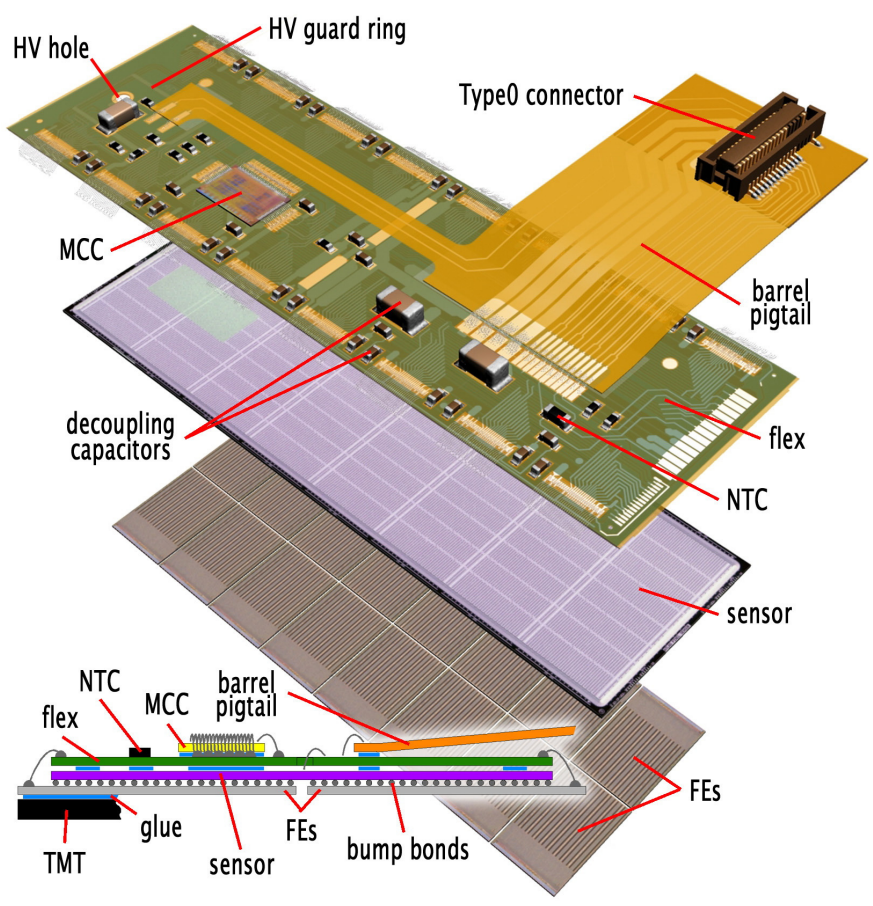

Fig. 2. The ATLAS Pixel Detector module [2].

The modules are designed to cope with a lifetime dose of $500 \mathrm{kGy}$.

\section{Readout}

The data connection to the Pixel Detector is realised using optical links. They connect the off-detector Read-Out Drivers (RODs) to the modules over a distance of $80 \mathrm{~m}$. Each module has one downlink (TTC) providing clock, trigger signals, detector calibration data and commands. These are encoded into a Bi-Phase Mark (BPM) signal which is transmitted at 40 MHz. The BPM signals are decoded using a Digital Optical Receiver Integrated Circuit (DORIC) located on an OptoBoard [5] before being transmitted via Low Voltage Differential Signals (LVDS) to the modules. The Module Control Chip (MCC) sends data from collision events to the data acquisition system outside the detector utilising Opto-Boards, where the signal is converted to an optical signal by verticalcavity surface-emitting lasers (VCSEL) arrays, so-called Txplugins. Data is then transferred off-detector from the modules to the RODs via a data-link whose speed and modularity depend on the location of the modules in the detector. Barrel Layer-2 modules have one data-link transmitting at $40 \mathrm{MHz}$ while the modules in the Disks and Barrel Layer-1 have one link which can run at either 40 or $80 \mathrm{MHz}$. The innermost barrel layer (B-layer) has, due to the high expected hit rate, two data-links per module which can each be read out at 80 $\mathrm{MHz}$ for an equivalent readout speed of $160 \mathrm{MHz}$. The layout of the pixel readout system from the signal generation in the sensor to the off-detector RODs can be found in Fig. 3.

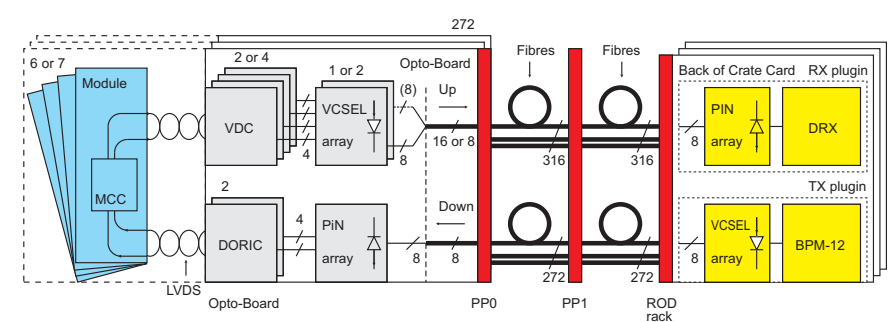

Fig. 3. Layout of pixel readout system [2].

\section{DETECTOR OPERATION}

\section{A. Detector calibration}

The working points for the readout systems and the modules themselves, including voltages and currents as well as temperature settings, must be checked on a regular basis to optimise the operation of the detector. In order to avoid data read-out errors, so-called optical scans are employed to ensure that the transmitting VCSELs for each link are sending light and also to determine stable sampling points for the datalinks in the laser-threshold/sampling-time phase space. Over the course of the last year the off-detector VECSEL arrays (Tx plugins) mentioned in Section II-C started to fail at a rate of 2-3 plugins per week. The reason for these failures is currently being investigated. Replacement of a Tx-plugin and readjustment of laser power settings for the affected OptoBoard requires about four hours during which no data can be taken. The overall timing for all modules that takes into account trigger delays, cable lengths and module-by-module variations has a dispersion of only $0.17 \mathrm{~ns}$.

Front-end electronics are also calibrated when necessary. The target thresholds for each pixel are 3500 electrons for the 2011 run period. Threshold and noise of the analog front-end are measured by injecting charges into the readout amplifiers in the vicinity of the expected threshold and counting the number of hits obtained for each injection. The response curve is then determined by fitting the distributions. To obtain homogenous thresholds for the whole detector, a so-called threshold scan is performed: the injected charge is fixed to the target threshold value and the discriminator threshold is varied to obtain a response fraction of about 50\% [6]. In Fig. 4a the measured threshold values of all pixels included in this scan are shown. The typical dispersion is 40 electrons. The noise per pixel, shown in Fig. $4 \mathrm{~b}$, amounts to 170 electrons for normal and long pixels, and 300 electrons for the $2.5 \%$ of all pixels where the electronics channels have two sensor pixels connected for seamless coverage in the short pixel direction (ganged pixels). About $0.3 \%$ of all pixels are masked for ATLAS data-taking to reduce the rate of noise to approximately $10^{-7} \mathrm{hits} / \mathrm{pixel} / \mathrm{event}$. Additionally, after datataking, another mask is applied that reduces the noise to less than $10^{-9}$ hits/pixel/event.

The ATLAS Pixel Detector has the capability of measuring the so-called Time over Threshold (ToT) of each pixel. This is the time for which the signal is above the threshold in units of bunch crossings $(\mathrm{BC}=25 \mathrm{~ns})$. This measurement is proportional to the deposited charge and results in improved 


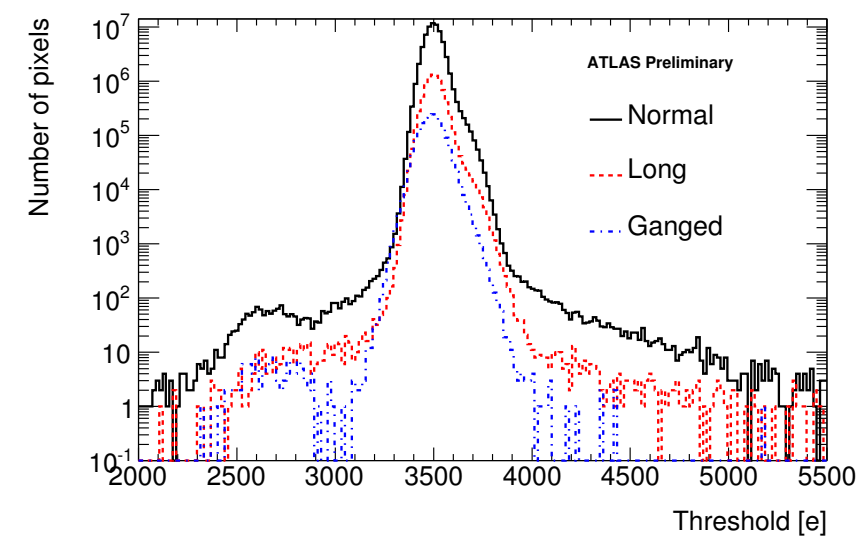

(a)

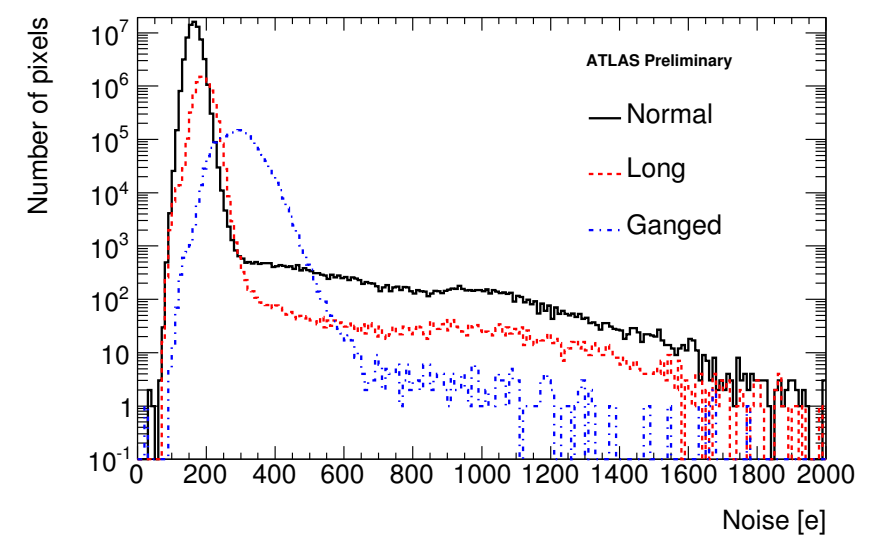

(b)

Fig. 4. Pixel threshold (a) and noise (b) distributions for all pixels included in the scan. The target threshold was 3500 electrons [7].

position resolution for particles that deposit charge in more than one pixel. Furthermore, the energy loss is proportional to the deposited charge and enables particle identification with the Pixel Detector using the energy loss per distance. The extracted energy loss per unit distance $(d E / d x)$ is shown in Fig. 5. The tuning target for ToT is $30 \mathrm{BC}$ for a charge of 20,000 electrons, which is the most probable value for a minimum ionising particle and has a resolution of $1 \mathrm{BC}$.

\section{B. Operation with beam}

The Pixel Detector is configured to remain in a safe standby state until the LHC has declared stable beam conditions. Standby means that the high voltage is off (sensors unbiased). This is enforced by a hardware interlock that is directly linked to the LHC status. An unbiased, undepleted sensor can, however, lead to a large number of noise hits per module which can block the data acquisition system. For this reason, the preamplifiers for each pixel are also switched off when the detector is in standby. Once the LHC declares stable beam conditions, the beam conditions and the collimator settings are checked by the ATLAS shift crew and the so-called warmstart is performed meaning that the high voltage is ramped up and the preamplifiers are switched on. While recording more than $5 \mathrm{fb}^{-1}$ during 2011, the data-taking efficiency of the Pixel Detector has been close to $100 \%$. Inefficiencies are

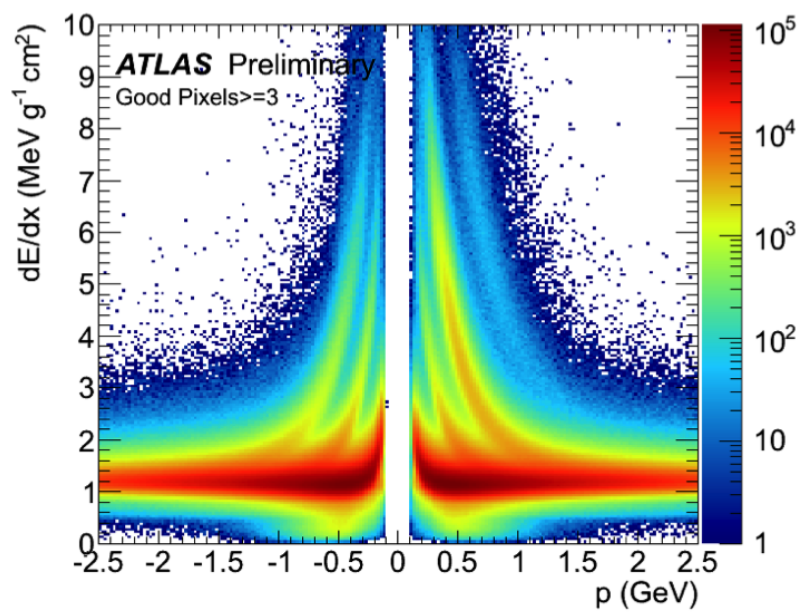

Fig. 5. Particle energy loss per distance $(d E / d x)$ for a measured momentum using the Pixel Detector ToT [7].

mainly due to the manual warm-start procedure which will be automated in the near future. Once LHC removes the stable beam conditions declaration, the Pixel Detector automatically goes back to standby.

\section{Detector Status}

After about one and a half years of very successful LHC and ATLAS running, $96.8 \%$ of the Pixel Detector is operational. Out of the 1792 modules, only 55 are disabled (3.2\%), mostly due to problems with communication, but also problems with the high voltage in one-third of all cases. A very small fraction $(0.17 \%)$ of the front-ends are disabled in addition. Other problems that are observed are pixels with disconnected bumps $(0.1 \%)$, mostly in the disk modules and pixels with defective analogue readout $(0.04 \%)$.

\section{A. Radiation damage}

Since the Pixel Detector is the ATLAS component that is closest to the colliding LHC beams, it is exposed to high radiation. During the first phase of LHC running, up to the year 2015, it is expected that the Pixel Detector will be irradiated to a fluence of $10^{15} \mathrm{neq} / \mathrm{cm}^{2}$. This will have an effect on the performance of the detector as well as the required working point. Therefore, the effects of radiation damage are measured on a regular basis and are used to tune Monte Carlo simulations of the detector.

\section{B. Leakage current measurements}

Irradiation of the modules results in an increase of the leakage currents, which can be measured with different methods. In order to measure the current per pixel, dedicated circuitry in the front-end chips can be employed. A significant increase of the leakage current was observed after the LHC had delivered an integrated luminosity of $1 \mathrm{fb}^{-1}$, first for the B-layer, the innermost layer, and later for the entire barrel region. Module leakage currents are measured by summing the currents for all pixels in a module and by using dedicated boards for leakage 


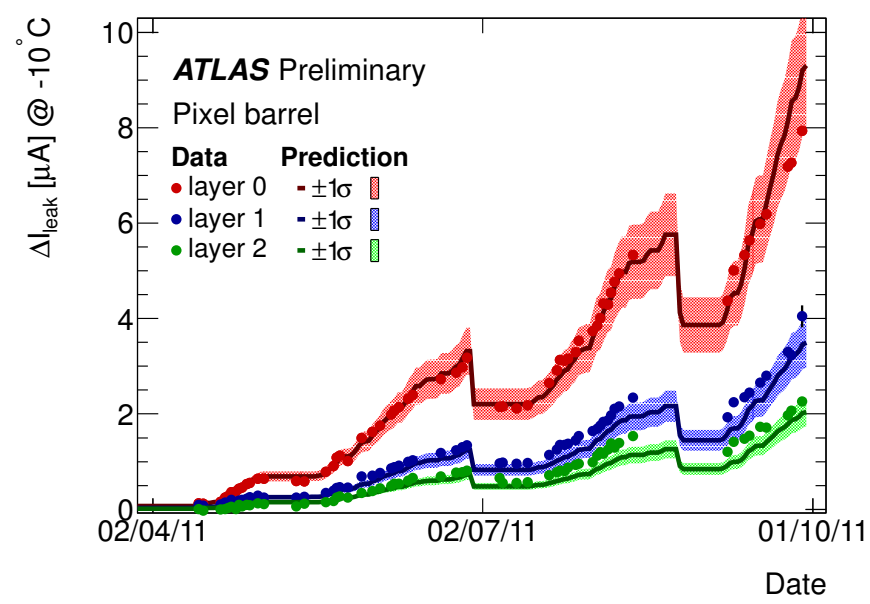

Fig. 6. Average increase in the leakage current per module as a function of date for the barrel layers. The measurement is compared to a model that is indicated by solid lines [7].

current measurements that are connected to a subset of high voltage lines in the detector. The leakage current, corrected to a common temperature of $-10^{\circ} \mathrm{C}$ for all modules, is shown as a function of time in Fig. 6 and compared with a model that predicts an increase of the leakage current proportional to the sensor volume and fluence. Good agreement is observed with this model.

\section{Depletion voltage measurements}

In addition to increasing leakage currents one expects a decrease in the voltage needed to fully deplete the sensors before type inversion and an increase in the needed voltage afterwards. Before type inversion, this effect is measured using so-called crosstalk scans across the detector. High charges are injected into the neighbouring pixels that will give a hit in the pixel at question if it not fully depleted. For each injection, the number of pixels with no hits is counted as a function of the bias voltage. The outcome of several such scans is shown in Fig. 7. It is observed that the depletion voltage has decreased as expected from June to August, but increased from June to July due to reverse annealing effects.

\section{CONCLUSION}

The ATLAS Pixel Detector is calibrated and performing well. Communication via optical links is well established with a timing dispersion between modules of only $0.17 \mathrm{~ns}$. The hit threshold for 2011 data-taking is tuned to 3500 electrons with a dispersion of 40 electrons. The Pixel Detector has the capability of measuring the Time over Threshold in addition to hits which is tuned to $30 \mathrm{BC}$ with a resolution of $1 \mathrm{BC}$. To increase data-taking efficiency while decreasing the number of people on shift automatic switch-on and -off procedures are planned. About $96.8 \%$ of the detector are operational. Radiation effects are already visible and agree well with expectations from detailed simulations.

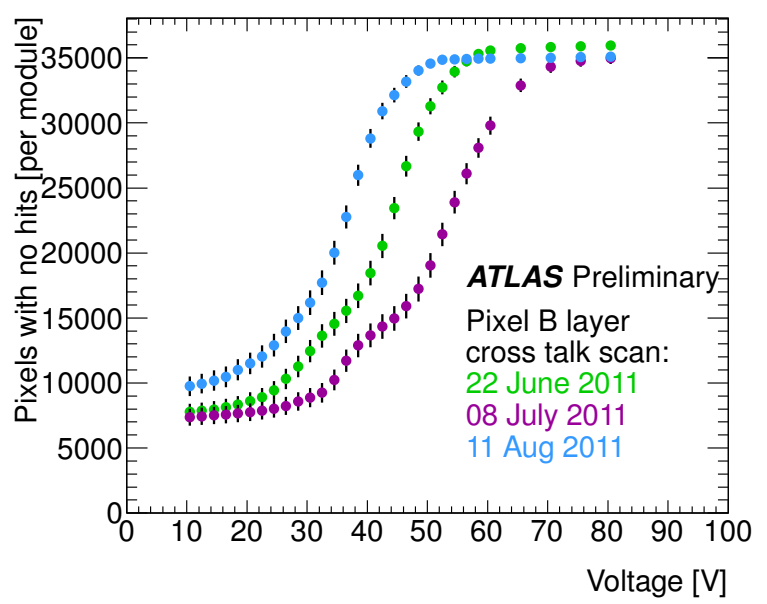

Fig. 7. Results of the various crosstalk scans as a function of the bias voltage in the sensor [7].

\section{REFERENCES}

[1] The ATLAS Collaboration, JINST 3 (2008) S08003.

[2] G. Aad et al., JINST 3 (2008) P07007.

[3] I. Peric et al., Nucl. Instrum. Meth. A 565 (2006) 178.

[4] The ATLAS Collaboration, CERN/LHCC/98-13, 1998.

[5] K.K. Gan et al., Nucl. Instrum. Meth. A 570 (2006) 292.

[6] The ATLAS Collaboration, EPJC 70 (2010) 787.

[7] https://twiki.cern.ch/twiki/bin/view/AtlasPublic/ApprovedPlotsPixel 